\section{Visual angle and apparent size of objects in peripheral vision}

\author{
L. R. NEWSOME \\ University of Queensland, St. Lucia, Queensland, Austrialia, 4067
}

Measurements of apparent size were obtained by distance adjustment of a peripherally viewed stimulus to produce a match to a foveally viewed standard. As eccentricity increased, the peripheral stimulus was adjusted at distances of progressively greater visual angle, indicating that a continuous diminution in apparent size occurs with increased eccentricity. This effect was found to be stable for several conditions of illumination and for changes in the light adaptive state of S. Apparent size diminution and apparent distance increase were also found for familiar objects viewed in an open field.

Scattered through the literature of psychology are reports that peripherally seen objects appear diminished in apparent size (Collier, 1931; Fraisse, Ehrlich, \& Vurpillot, 1956; Grindley, 1930; Helmholtz, 1962 ; James, 1890 ; Piaget, Rutschmann, \& Matalon, 1959; Salaman, 1929), and two papers have attempted to explain visual illusions in terms of spatial anisotropies of the peripheral visual field (Pearce \& Taylor, 1962; Richards \& Miller, 1971). In spite of the frequent reference to the diminishment effect, there is no clear indication of either its magnitude or how magnitude varies with viewing eccentricity. Stevens (1908) tried to obtain measurement of the apparent size decrease by using simultaneous comparisons of a peripheral stimulus with a fixed stimulus in foveal view but obtained inconclusive results.

In the present study, the apparent size of a peripheral object is measured by manipulation of the distance of the peripheral stimulus until it matches the apparent size of a foveally viewed standard. The technique of changing visual angle by adjusting the distance of the stimulus has been employed by other investigators (e.g., Thouless, 1931; Joynson, 1949), and indications were obtained from a pilot study that reliable measurements could be obtained with peripheral viewing.

The purpose of the first experiment was to provide measurements of apparent extent (size) for a number of eccentric positions across the visual field. Subsequent experiments explored the effect of varying illumination conditions on such measurements and the relationship of apparent size to judgments of apparent distance.

\section{EXPERIMENT 1} Method

Subjects. Ten male students from the University of Queensland volunteered to serve as $\mathrm{Ss}_{5}$. These were selected as being right-eye dominant and were screened for defects in visual acuity, amplitude of accommodation, and ametropia.

Apparatus. The stimulus objects were two $7.3-\mathrm{cm}$ squares of matte black material, displayed to appear as equilateral diamonds. One square served as the peripheral stimulus and was attached to the top of a 30-cm-high thin metal rod. The rod was carried by a sliding block on a track mechanism. A $150-\mathrm{cm}$-long arm carrying the track mechanism was pivoted to the center of one edge of a large table so that the arm lay across the table. Above the pivot was a headrest and temple clamp arrangement. When an $S$ was seated so that his right eye was directly above the pivot, the peripheral stimulus could be set by $E$ to any point across S's field of view on a horizontal plane level with the eye. A smooth perspex handwheel mounted horizontally above the pivot and below S's chin allowed $S$ to adjust the distance of the peripheral stimulus through a range of $130 \mathrm{~cm}$ to within $15 \mathrm{~cm}$ of his eye. To promote kinesthetic position confusion, movement of the handwheel was by finger friction to the top surface of the wheel.

The second black square served as a standard stimulus and was attached to a bar projecting out from a white semicircular background screen. The standard stimulus was located at a distance of $100 \mathrm{~cm}$ from S's right eye and directly in front of it. The background screen was of $155-\mathrm{cm}$ radius, was $65 \mathrm{~cm}$ high, and extended across the full extent of $S$ 's visual field. The screen was illuminated uniformly to have a luminance of $0.21 \mathrm{~cd} / \mathrm{m}^{2}$. An occluding shield was provided to cover S's left eye. A representation of the apparatus is given in Fig. 1.

Procedure. Each $\mathbf{S}$ was given six trials with the peripheral stimulus set at $80,70,60,50,40,30,20$, and $10 \mathrm{deg}$ temporally (right visual field) and $10,20,30$, and 40 deg nasally. On half of these trials, the peripheral stimulus was presented at a distance of $150 \mathrm{~cm}$ from $S$ 's eye. For the remaining trials, the initial setting was $30 \mathrm{~cm}$. Both the peripheral angle and the initial setting were determined from a random order, a new order being used for each $\mathrm{S}$. A trial consisted of S's adjusting the peripheral stimulus in or out from the initial setting until it reached an apparent equivalence

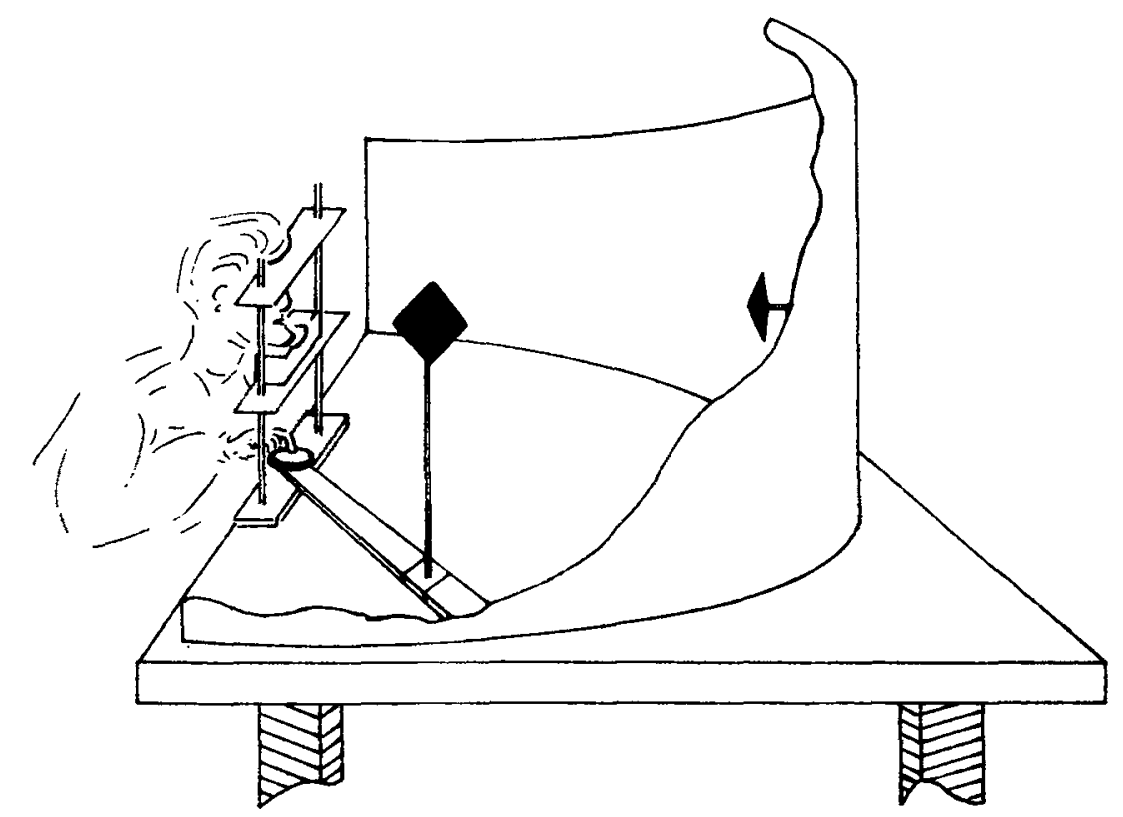

Fig. 1. Apparatus used to provide a simultaneous display of two stimulus squares with one stimulus adjustable in distance and eccentricity. 


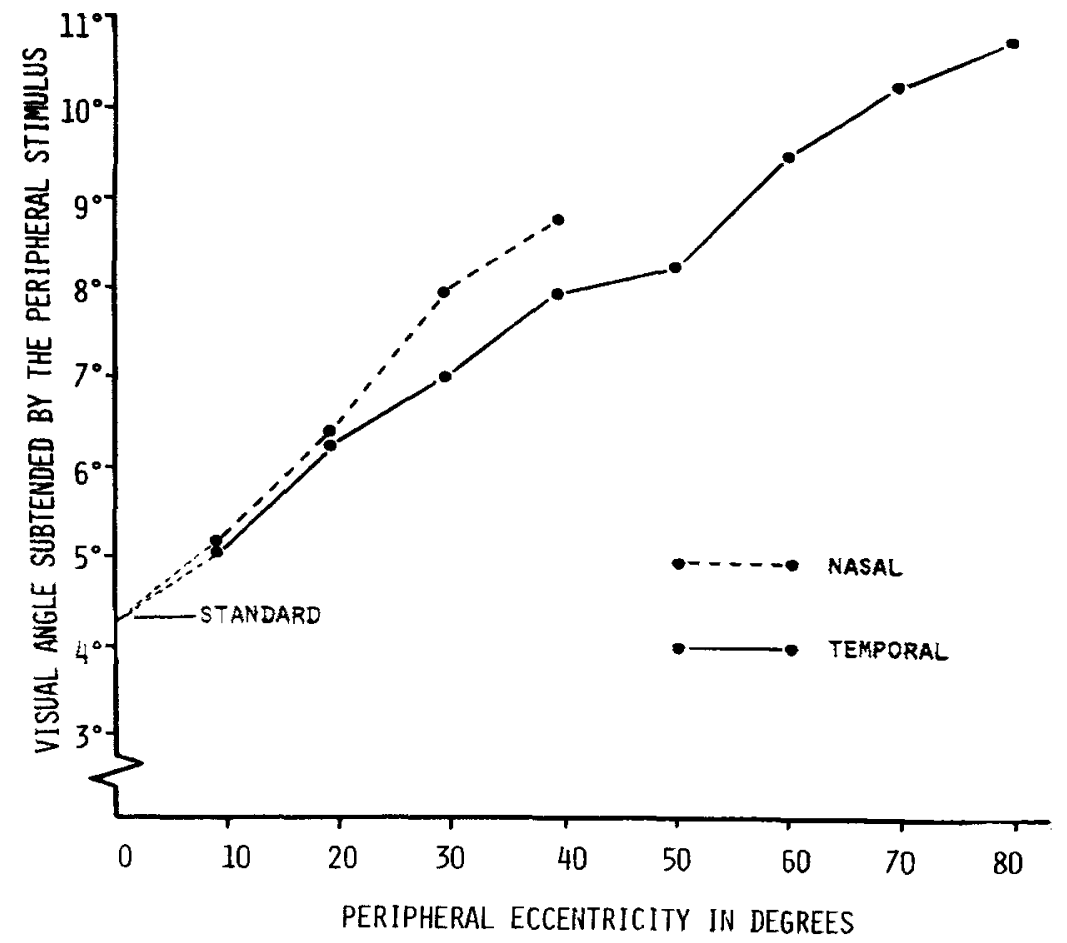

Fig. 2. Mean visual angle subtended by the peripheral stimulus at the point of subjective size equality.

with the standard stimulus. $S$ was instructed to maintain fixation of the center square (the standard stimulus) throughout each trial and to adjust the other square until it "appeared the same size" as the center square.

Twelve preliminary trials were given, and $\mathrm{S}$ was shown how to find the PSE by moving the peripheral stimulus back and forth through the zone of uncertainty. A check on the maintenance of fixation was made on each trial by $\mathrm{E}$ by sighting along the edge of the apparatus just outside S's field of vision. It was found that even rapid saccadic movements could easily be detected, and if such movements occurred during preliminary trials, $S$ was admonished. Subsequent trials observed appeared generally to be free of detectable eye movements. During the initial instruction period, no attempt was made to hide the apparatus from S's view and $S$ was allowed ad lib inspection.

Measurements of the distance of the adjustable stimulus from S's eye were taken after each setting. These were transformed to give the visual angle achieved relative to that made by the fixated central stimulus.

\section{Results and Discussion}

Figure 2 shows the mean visual angle necessary for equal apparent extent in the two stimuli to be achieved when the adjustable stimulus was displayed at different locations in peripheral vision. An analysis of variance indicated that a progressive increase in visual angle was required with increase in eccentricity $[F(11,9)$ $=20.9, p<.01]$. The effect of starting position was significant $[F(1,9)=$ $17.3, \mathrm{p}<.01 \mathrm{l}$, but the interaction between angle and starting position was not $(F<1)$. Examination of the results for individual $S s$ revealed that the starting position effect can be attributed to measures given by 4 of the $10 \mathrm{Ss}$.

Although Fig. 2 adequately represents the overall trend in settings as a function of eccentricity, there were wide individual trend variations. For instance, for $80 \mathrm{deg}$, the most extreme eccentricity, individual means ranged from 21.3 to $55.5 \mathrm{~cm}$. However, the distribution of settings for any given individual was relatively smaller in range and did not increase markedly with eccentricity, the SD ranging from $5,3 \mathrm{~cm}$ (approximately $0.5 \mathrm{deg}$ in terms of visual angle) to $6.7 \mathrm{~cm}$ (1.7 deg) for the temporal visual field and from $5.4 \mathrm{~cm}(0.6 \mathrm{deg})$ to $6.2 \mathrm{~cm}(0.9 \mathrm{deg})$ for the nasal field in order of increasing eccentricity.

The extent of the main effect demonstrates that a peripherally viewed object must be placed so as to achieve a greater visual angle than when viewed directly to appear the same in extent. Alternatively, these results may be interpreted as showing that, for a constant object size and distance, viz, a constant visual angle, a stimulus object appears progressively diminished in size as it is moved farther out to the periphery of vision. This agrees with the earlier reports (James, 1890; etc.) that peripheral objects appear diminished in size. The present results, however, also demonstrate the magnitude of the effect and show that the effect increases with increased eccentricity of viewing.

\section{EXPERIMENT 2}

In Experiment 1, although features of the environment were kept constant, it is possible that at least part of the observed effect was due to the use by $S$ of the juxtaposition of the movable stimulus object with extraneous background features such as the surface texture of the background materials. It is known that, with foveal vision, size judgments often change markediy when contextual and background information is removed. The present experiment tests this possibility for the peripheral case by comparing measurements obtained under the conditions of Experiment 1 with similar measurements taken with the use of luminous stimuli in an otherwise dark room.

\section{METHOD}

Subjects. Ss were seven male students selected for normal eyesight.

Apparatus. The apparatus of Experiment 1 was modified such that the background was black and the two stimulus squares comprised $7.6-\mathrm{cm}$ squares of greenish-white luminescent material. In the absence of other illumination, these squares had a luminance of $1.1 \times 10^{-4} \mathrm{~cd} / \mathrm{m}^{2}$.

Procedure. Procedure was similar to that of Experiment 1. Trials were restricted to the angles of $80,60,40$, and $20 \mathrm{deg}$ temporally and 20 and 40 deg nasally for right eye only, and two trials were given per angle for each condition. All trials started with the peripheral stimulus positioned $30 \mathrm{~cm}$ from S's eye. The first set of 12 experimental trials was given under normal room illumination conditions, the stimulus squares appearing greenish-white against the black background. After a 30-min dark adaptation period, $S$ was then given an additional 12 trials using the luminous squares in otherwise total darkness.

\section{Results}

No significant difference was observed between the illumination and nonillumination conditions $[F(1,6)=$ $2.45, p>.05]$, but, as in Experiment 1 , an analysis of variance 
of the data showed that eccentricity was again a significant factor in determining set distance $[F(5,30)=$ $5.87, p<.01]$. It may therefore be concluded that background and contextual information played no part in determining the results found in Experiment 1.

\section{EXPERIMENT 3}

One of the more immediate explanations of the results of Experiment 1 is that the increase in visual angle required to maintain the peripheral stimulus at constant apparent size is a function of the diminished, or impoverished, quality of peripheral vision. It has been shown that the apparent size of an object may be decreased, and the apparent distance increased, by either dimming the stimulus or reducing its contrast to the ground (Johns \& Sumner, 1948). The impoverishment explanation assumes that the effect of peripheral viewing is similar to a direct dimming and reduction in contrast of the stimulus. Experiment 3 offers a test of the impoverishment hypothesis by comparing results obtained under photopic conditions with those obtained under scotopic conditions. Crawford (1937) has shown that there are differential changes of foveal and peripheral retinal sensitivities between light-adapted and dark-adapted conditions. Mandelbaum and Sloan (1947) have shown that such differential changes occur also for visual acuity. If perception of apparent size relates to the sensitivity and acuity of peripheral vision, factors that correlate with dimmed and impoverished quality of the viewed object, then measurements obtained under scotopic conditions should differ from those obtained under photopic conditions.

\section{Method}

Subjects. These were five male students selected for normal uncorrected vision.

Apparatus and procedure. The apparatus was the same as for the previous experiment, except that under the scotopic condition, both stimuli were covered with heavily exposed and developed photographic negative. When covered, neither stimulus was visible to a number of observers unless the observer was almost completely dark-adapted. Measurements of the actual light output of these stimuli could not be obtained. All Ss were given the same procedure as for Experiment 2 under the illuminated condition first and then dark-adapted for $30 \mathrm{~min}$ before being given the remaining set of trials for the scotopic condition. In practice, it was found necessary to use a slightly

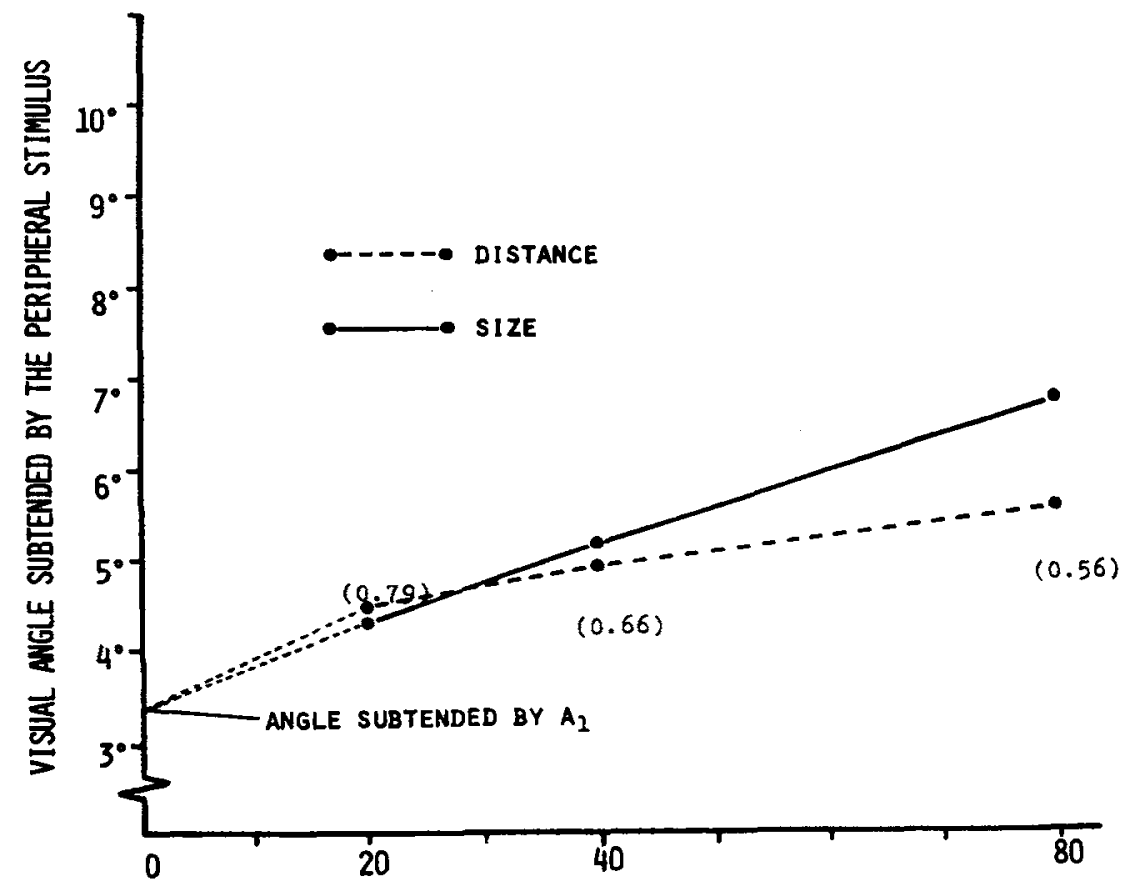

PERIPHERAL ECCENTRICITY IN DEGREES

Fig. 3. Mean visual angle subtended by $A_{2}$ at the PSE with $A_{1}$. Relative percentage distance of $\mathbf{A}_{2}$ for the "equal distance" condition is given in parentheses for each eccentricity.

less dense filter for the standard stimulus in order to prevent its disappearance due to the lower sensitivity of the fovea.

\section{Results and Discussion}

There was no observable difference between photopic and scotopic conditions $(\mathrm{F}<1)$, although the angle effect was still apparent as in the two previous experiments $[F(5,20)=4.67$, $p<.01]$. This experiment was thus unable to demonstrate that adjustments could be influenced by an induced change in retinal sensitivity. By reason of previous argument, it is concluded that judged extensivity of the peripheral stimulus is not simply a function of decline in nonfoveal, retinal sensitivity.

\section{EXPERIMENT 4}

In the previous experiments, it has been demonstrated that abstract stimulus objects can appear diminished in size with peripheral viewing, but it has yet to be demonstrated that the same holds true for normal objects in a familiar environment. The present experiment attacked the problem by using two men as stimulus objects in the context of a university playing field. Added to a number of trials where $S$ was required to judge when the two men appeared equal in extent was a set of trials in which $S$ was required to equate apparent distances.
The latter instructional condition was included to provide information on the ability of an $S$ to judge the distance of an object using only peripheral vision.

\section{Method}

Subjects. Ten male students with normal and unaided vision volunteered for this experiment.

Procedure. S $w$ as positioned standing in the middle of a large playing field facing the back of a 167-cm-tall (5 ft, 6 in.) male assistant $\left(A_{1}\right)$ standing $30.6 \mathrm{~m}$ (100 ft) away. Each trial began with a second assistant of equal height $\left(A_{2}\right)$ walking either towards or away from $S$ along one of three paths radiating from $S$ 's position. These paths took the angles 20,40 , and 80 deg temporally to $S$ 's facing direction. $S$ was allowed binocular viewing and was told to fixate the center of $A_{1}$ 's back. S's task was to call to $A_{2}$ to stop when, according to whichever condition prevailed, $A_{2}$ appeared to be of the same "size" at the same "distance" as $A_{1}$. In between trials, $S$ was told to look down at the ground until $E$ indicated readiness for the next trial to begin. As before, no further explanation was given to $\mathbf{S}$ as to what was meant by "size" or "distance." Half of the Ss were given the "distance" task first, and for the other half the "size" task came first. On 
alternative trials, $A_{2}$ started either 6 or $50 \mathrm{~m}$ away from $\Omega$ and moved either away from or towards $S$ accordingly. Two trials only were given for each angle and each $S$.

Measurements of the distance from $S$ at which a halt was called were taken by $A_{2}$ from a steel tape laid alongside the paths and relayed via transceiver to an off-field data recorder. E stood immediately behind and to the offside of $S$ in order to monitor the proceedings and to give appropriate instructions.

Results and Discussion

Figure 3 shows the mean visual angle at which $A_{2}$ was judged "equal to $A_{1}$ " for both "size" and "distance" conditions. Distances relative to the standard distance of $A_{1}$ are shown in parentheses for the "distance" condition. An analysis of variance on the data obtained indicated that there was no difference overall between the size and distance tasks $[F(1,9)=1.24$, $\mathrm{p}<.95$ ] but that significant Task by $\mathrm{S}$ and Angles by $S$ interactions both occurred $[F(9,60)=6.62, p<.01 ;$ and $F(18,60)=2.10, p<.05$, respectively $]$. The angles' main effect was also significant, as would be expected from the trends in Fig. 2 $[F(2,18)=25.6, p<.01]$. Mean variability of set visual angle did not vary markedly with increased eccentricity, the SD in terms of visual angle ranging from 0.49 to $0.76 \mathrm{deg}$ for the size task and 0.11 to $0.13 \mathrm{deg}$ for the distance task.

Inspection of the raw data revealed the probable source of both interactive effects. In the "size" task, a numbe; of Ss found it necessary, at the most extreme peripheral angle, to allow $\mathbf{A}_{2}$ to recede or approach to within about $8 \mathrm{~m}$ of themselves in order to obtain the appropriate perceptual equivalence. In the "distance" task, in no instance was $A_{2}$ told to stop at points closer than $13 \mathrm{~m}$. Data from those Ss who were able to obtain a satisfactory "size" match at $15 \mathrm{~m}$ or more showed little difference between "size" data and "distance" data. It is suggested here that in the case of the "distance" task, other cues to distance tended to predominate when $A_{2}$ was closer than about $15 \mathrm{~m}$. S would have known without reference to the extensivity of the proximal stimulus that $A_{2}$ was too close to equal the distance of $A_{1}$. This factor, then, could account for the attenuated data range for the "distance" task at the angle of $80 \mathrm{deg}$ and the subsequent interaction effects.

The lack of difference between tasks, apart from the interactive effects previously discussed, suggests that the same perceptual attributes of the stimuli were used for both types of task. Furthermore, the close resemblance of the data trends here to the trends found in the previous experiments suggests that a common phenomenon is being exemplified by all four experiments. The compelling conclusions from the present experiment are that an illusory diminution of extensivity can occur with peripheral viewing of real objects in the context of a normal visual environment, and that this may be coupled with an increase of the apparent distance of the object.

\section{GENERAL DISCUSSION}

All four experiments offer evidence that there is a reduction in apparent size of objects when viewing becomes peripheral, and that the reduction continues monotonically with increased eccentricity. The results of Experiment 4 indicate that visual field anisotropies occur for perceived distance also.

Although the anisotropic effects demonstrated by these experiments were large, there are some reservations to be stated about the data and the method used.

First, while eye movements were monitored in the first experiment, no objective record of eye position was possible to guarantee that undetected movements did not occur. In view of the present results, the most likely effect of the occurrence of eye movements would be to reduce the influence of peripheral diminution on the settings made. This suggests that the magnitude of the anisotropies indicated could be conservative.

A second reservation is that with the method used for varying visual angle, there is no control of the possibly perturbing effects of distance judgments independent of visual angle. This applies to Experiment 1 as well as to Experiment 4. Further work is required where visual angle is varied directly while distance is held constant.

Exploration of the peripheral field other than the horizontal plane portion is also required. Until the parameters of peripheral change in apparent size have been more thoroughly researched, it is perhaps premature to attempt an extensive explanation of the effect. However, several tentative explanations can be discussed.

An immediate suggestion is the impoverishment hypothesis already discussed in Experiment 3. The results of Experiment 3 failed to indicate changes in perception with conditions inducing changes in stimulus impoverishment. The supposition made in Experiment 3, that lowered sensitivity and acuity is the same in effect as impoverishment by dimming, is a tenuous one. But unless objective specifications can be attached to what is meant by impoverishment in peripheral vision, the impoverishment hypothesis adds nothing by way of explanation except a semantic translation in description. Another difficulty with this hypothesis is to say which is cause and which is effect. Does a peripheral object look smaller because it appears vague or dimmed, or does the object look dimmed because it looks smaller?

A second approach to explaining the effect might be to relate apparent size to the structural properties of the eye. This has some attractiveness, since the untransformed results (inverse of data in Fig. 2) of Experiment 1 plotted as a function of eccentricity bear some resemblance to the decline in retinal receptor density as provided by Dsterburg (1935).

A second structural explanation is that the peripheral decline in apparent size follows the diminished image size that results from the reduced distance of the peripheral retina from the lens. The optical explanation is important to the density theory in that it poses the problem of defining the relationship between the stimulus field and the topography of the retina: there is yet no clear specification of the relationship. Both structural explanations suffer the same difficulty. There is no apparent reason why perceived size should be isomorphic with either receptor topography or the geometrical dimension of the retinal image.

A further feature of the visual system of possible relevance to the present data is the mechanism concerned with the generation of the binocular horopter, the locus of binocularly perceived points in space of common depth. The horopter defines a concave surface having something of the same shape across the horizontal plane as the curve for the raw distance data of the present experiments. A concise definition of a causal relationship between the two curves would be difficult, as the horopter refers to conditions of binocular fixation of the stimulus object. The practical horopter must also remain confined to within about $40 \mathrm{deg}$ of the midfrontal plane. While it may still be possible that the locus of equal distance points follows the horopter when viewing is monocular and peripheral, and dictates the points of isotropic size, the continued decline in setting distances shown by the present data for points considerably outside the 40-deg limits remains unaccountable in these terms.

None of the explanations provides a satisfactory and compelling reason why visual field anisotropies for 
apparent size should occur. Nor do they suggest how these anisotropies affect the adaptive function of the visual system. Further knowledge of the parameters of peripheral size perception may be required before a comprehensive explanation of such anisotropies can be formulated.

\section{REFERENCES}

COLLIER, R. M. An experimental study of form perception in peripheral vision. Journal of Comparative Psychology 1931, 1, 281-289.

CRAWFORD, B. H. The luminous efficiency of light entering the eye pupil at different points and its relation to brightness threshold measurements. Proceedings of the Royal Society, B. 1937, 124, 81-96.

FRAISSE, P., EHRLICH, S., \& VURPILLOT, E. Etudes de la centration perceptive par la méthode tachistoscopique. Archives de Psychologie, 1956, 35, 194-214.
GRINDLEY, G. C. Psychological factors in peripheral vision. Medical Research Council, Special Reporting Service, No. 163,1931

HELMHOLTZ $\mathrm{H} \mathrm{L} F$ Treatise in physiological optics. Vol. 1. (Trans. from the 3rd German ed., J. P. C. Southall) New York: Dover, 1962 .

JAMES, W. Principles of psychology. Vol. 2. London: Macmillan, 1890

JOHNS, E. H., \& SUMNER, F. C. The relation of the brightness differences of colours to their apparent distances. Journal of Psychology, 1948, 26, 25-29.

JOYNSON, R. B. The problem of size and distance. Quarterly Journal of Psy chology, 1949, 1, 119-135.

MANDELBAUM, A. J., \& SLOAN, L. L Peripheral visual acuity. American Joumal of Ophthalmology, 1947, 30, $581-587$.

PSTERBURG, G. Topography of the layer of rods and cones in the human retina. Acta Ophthalmologia, 1935 (Suppl. 61) 1-102.

PEARCE, D. G.. \& TAYLOR, M. N. Visual length as a function of orientation at four retinal positions. Perceptual \& Motor Skills, 1962, 14, 431-438.

PIAGET, J. The mechanisms of perception (Trans. from the French ed. $G$. $N$. Seagrim) London: Routledge \& Kegan Paul, 1969 .

PIAGET, J., RUTSCHMANN, J., \& MATALON," B. Nouvelles mesures des effets de centration en presentation tachistoscopique. Archives de Psychologie, 1959, 37, 140-165.

RICHARDS, W., \& MILLER, J. F. The corridor iliusion. Perception \& Psychophysics, 1971, 9, 421-423.

SALAMAN, $M$. Some experiments on peripheral vision. Medical Research Council, Special Reporting Service, 1929 , No. 136.

STEVENS, H. C. Paxticularities of peripheral vision. Psychological Review, $1908,15,69-93$

THOULESS, R. H. Phenomenal regression to the real object. British Journal of Psychology, 1931, 21, 338-359.

(Accepted for publication May 17, 1972.) 